\title{
The First Korean Case of NUP98-NSD1 and a Novel SNRK-ETV6 Fusion in a Pediatric Therapy-related Acute Myeloid Leukemia Patient Detected by Targeted RNA Sequencing
}

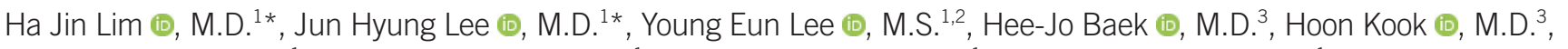

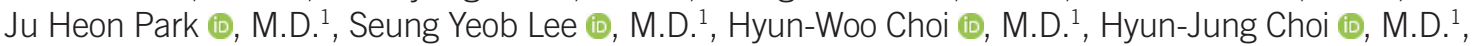
Seung-Jung Kee $\mathbb{C}^{\circ}$, M.D. $^{1}$, Jong Hee Shin $\mathbb{1}^{\circ}$, M.D. $^{1}$, and Myung Geun Shin $\mathbb{1}$, M.D. ${ }^{1,2}$

${ }^{1}$ Department of Laboratory Medicine, Chonnam National University Medical School and Chonnam National University Hwasun Hospital, Hwasun, Korea; ${ }^{2}$ Brain Korea 21 Plus Project, Chonnam National University Medical School, Gwangju, Korea; ${ }^{3}$ Department of Pediatrics, Chonnam National University Medical School and Chonnam National University Hwasun Hospital, Hwasun, Korea

\section{Dear Editor,}

Targeted RNA-sequencing (RNA-seq) using next-generation sequencing (NGS) technology is a highly accurate method for selecting and sequencing specific transcripts of interest [1]. We routinely applied a customized targeted RNA-seq system during the diagnostic phase of hematologic malignancies. Our system detected the first Korean case of NUP98-NSD1 and a novel SNRK-ETV6 fusion with therapy-related acute myeloid leukemia (t-AML) showing a dismal clinical course. NUP98-NSD1 accounts for approximately $4 \%$ of pediatric AML cases and shows a poor prognosis [2, 3]. It could be created by a cryptic t(5;11)(q35;p15.5) and exerts a leukemogenic function by binding near the HOX locus and MEIS1 to increase expression via histone modifications [4]. The Institutional Review Board of Chonnam National University Hwasun Hospital (CNUHH), Hwasun, Korea (CNUHH2020-091) approved this study and granted a waiver of consent due to its retrospective nature. This report highlights the role of high-throughput parallel targeted RNA-seq in enhancing the diagnostic yield of hematologic malignancies.
In April 2020, a 14-year-old girl visited the outpatient clinic of CNUHH 1.5 years and 1.9 years after a matched unrelated peripheral blood stem cell transplantation and initial diagnosis of $A M L$, respectively, for a follow-up bone marrow (BM) examination At initial diagnosis, the Korean AML 2012 regimen (doubleinduction strategy with idarubicin or mitoxantrone plus cytarabine, followed by consolidation therapy with cytarabine and etoposide) was administered and complete remission was achieved 28 days after the second induction. The laboratory findings showed a leukocyte count of $3.1 \times 10^{9} / \mathrm{L}$, absolute neutrophil count of $0.58 \times 10^{9} / \mathrm{L}$, hemoglobin of $114 \mathrm{~g} / \mathrm{L}$, and platelet count of $37 \times$ $10 \%$ L. BM aspirates revealed $28 \%$ leukemic blasts corresponding to French-American-British (FAB) type M2. The BM karyotype was 45,XX,add(3)(p25), del(5)(q?),-12,add(12)(p13)[8]//46, $X Y[12]$, and the multiplex reverse transcription (RT)-PCR (HemaVision kit; DNA Technology, Aarhus, Denmark) finding was negative.

Targeted RNA-seq (HEMEaccuTest RNA; NGeneBio, Seoul, Korea) of the BM sample using STAR-Fusion (ver 1.8.1) and
Received: June 29, 2020

Revision received: October 5, 2020

Accepted: January 4, 2021

Corresponding author: Myung-Geun Shin, M.D.

Department of Laboratory Medicine, Chonnam National University Hwasun Hospital, 322 Seoyang-ro, Hwasun-eup, Hwasun-gun, Jeollanam-do 58128, Korea

Tel: +82-61-379-7950, Fax: +82-61-379-7984,

E-mail:mgshin@chonnam.ac.kr

*These authors equally contributed to this study. 
FusionCatcher (ver 1.20) revealed NUP98-NSD1 and a novel SNRK-ETV6 fusion, which were confirmed by direct sequencing (Fig. 1). DESeq2 (ver 1.18.1) analysis showed that WT1, ERG, and BAALC expression increased 7.1, 5.6, and 4.1- $\log _{2}-$ fold, respectively, compared with 14 normal controls (Table 1). An additional tier II variant of WT1, NM_024426.3:c.1142C > A (p.Ser381*), and three tier III variants were detected by FreeBayes (ver 1.3.1) [5]. Further targeted DNA NGS (HEMEaccuTest DNA) confirmed the variants in targeted RNA-seq and additionally detected a tier II variant of KRAS, NM_033360.4:c.38G > A (p.Gly13Asp), and five tier III variants. However, no significant variant of FLT3, including FLT3-ITD, was detected. Donor lymphocyte infusion (DLI) was conducted on day 7 after the diagnosis; however, the BM blasts increased to $88 \%$ on day 29. The combination of fludarabine, cytarabine, idarubicin, and granulocyte colony-stimulating factor chemotherapy was started on day 35 and the BM blasts decreased to $<5 \%$ on day 71 with sustained thrombocytopenia; however, the condition repeatedly relapsed on day 134 and the patient expired on day 223.

NUP98-NSD1+ AML is characterized by frequent FAB-type
M4/M5, a normal karyotype, and HOXA/B upregulation [2]. Further, NUP98-NSD1 is mutually exclusive with other type II variants, but often co-occurs with type I variants such as FLT3-ITD or WT1 variants [2, 3]. FLT3-ITD is the most common variant in NUP98-NSD1+ AML (unlike our case), and its prognosis is dismal. Recent studies showed the promising therapeutic effects of dasatinib and navitoclax combination therapy and preemptive DLI based on minimal residual disease for NUP98-NSD1+/FLT3ITD+ AML $[6,7]$. Regarding the novel SNRK-ETV6 fusion, the defect in ETV6 is pathogenic in hematologic malignancies caused by rearrangement or deletions [8]. However, the partner SNRK gene defect at 3p22.1 has rarely been studied in hematologic malignancies but reportedly impacts hematopoietic cell proliferation and differentiation [9]. Further studies are needed to clarify the role of this novel fusion. This case also meets the criteria of t-AML, representing del( $5 q)$ with a complex karyotype and prior cytotoxic chemotherapy; both NUP98- and ETV6- rearrangements were reported in t-AMLs [10]. Additionally, the patient has a variant in TP53 (rs1042522), known to increase the risk of developing therapy-related myeloid neoplasms. Owing to
(4)

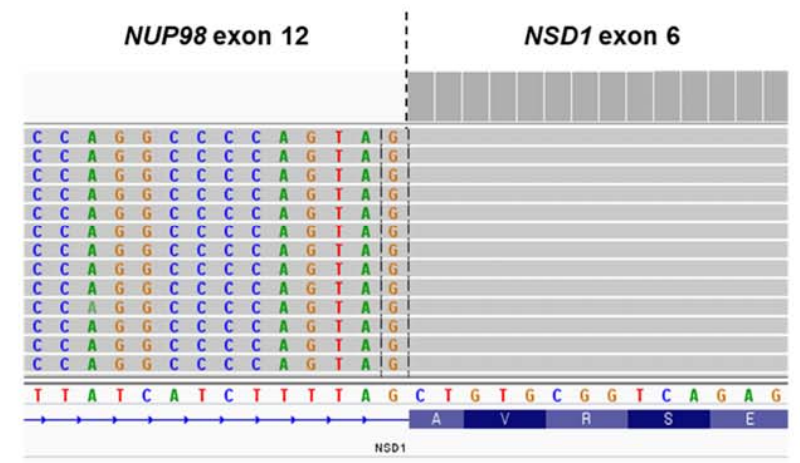

B

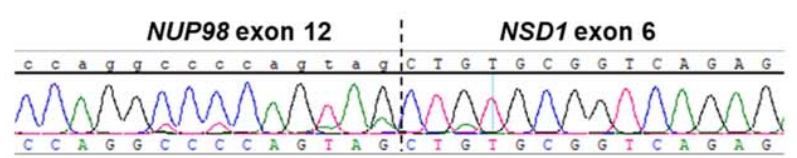

$\boldsymbol{0}$

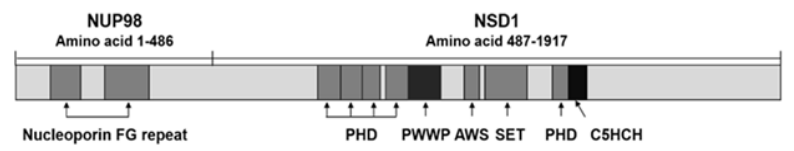

(0)

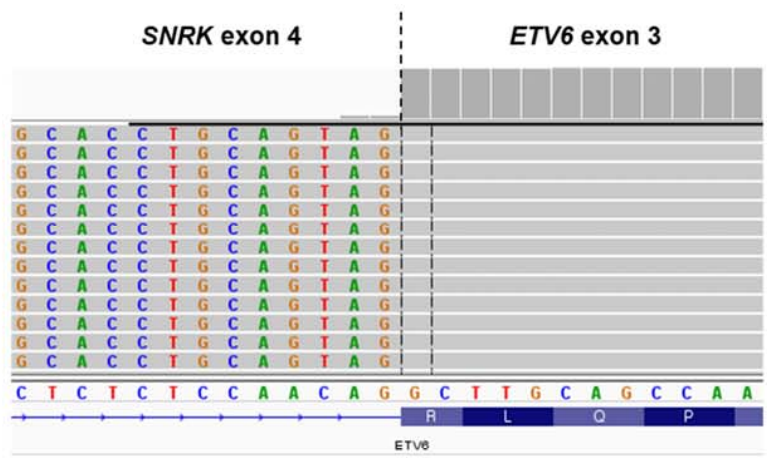

E
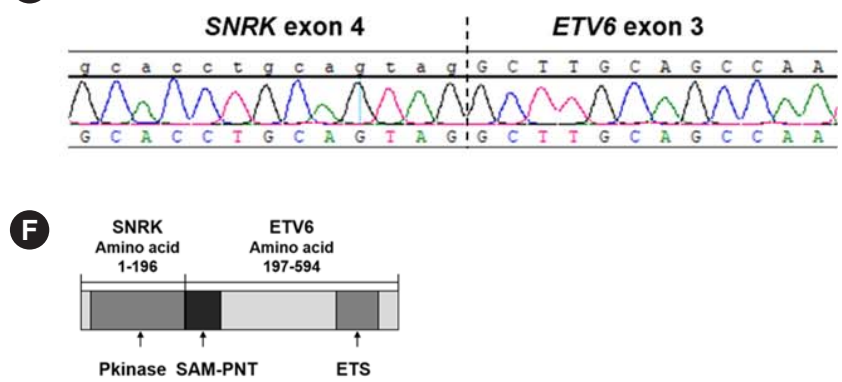

Fig. 1. Schematic representation of the NUP98-NSD1 (A-C) and novel SNRK-ETV6 (D-F) gene fusions and proteins. (A) Integrative genomics viewer (IGV) image showing the NUP98-NSD1 breakpoints with 171 supporting junction read counts. (B) Direct sequencing confirmed the identical breakpoint causing an in-frame fusion of NUP98-NSD1. (C) The predicted fusion protein translated from the NUP98NSD1 transcript based on a merged sequence produced by STAR-Fusion (ver 1.8.1), which contains domains similar to a previous report [2] but is shorter. (D) IGV image showing the novel SNRK-ETV6 fusion breakpoints with 484 supporting junction read counts. (E) Direct sequencing confirmed the identical breakpoint causing a novel in-frame fusion of SNRK-ETV6. (F) The predicted fusion protein translated from the SNRK-ETV6 transcript based on the merged sequence produced by STAR-Fusion (ver 1.8.1). 


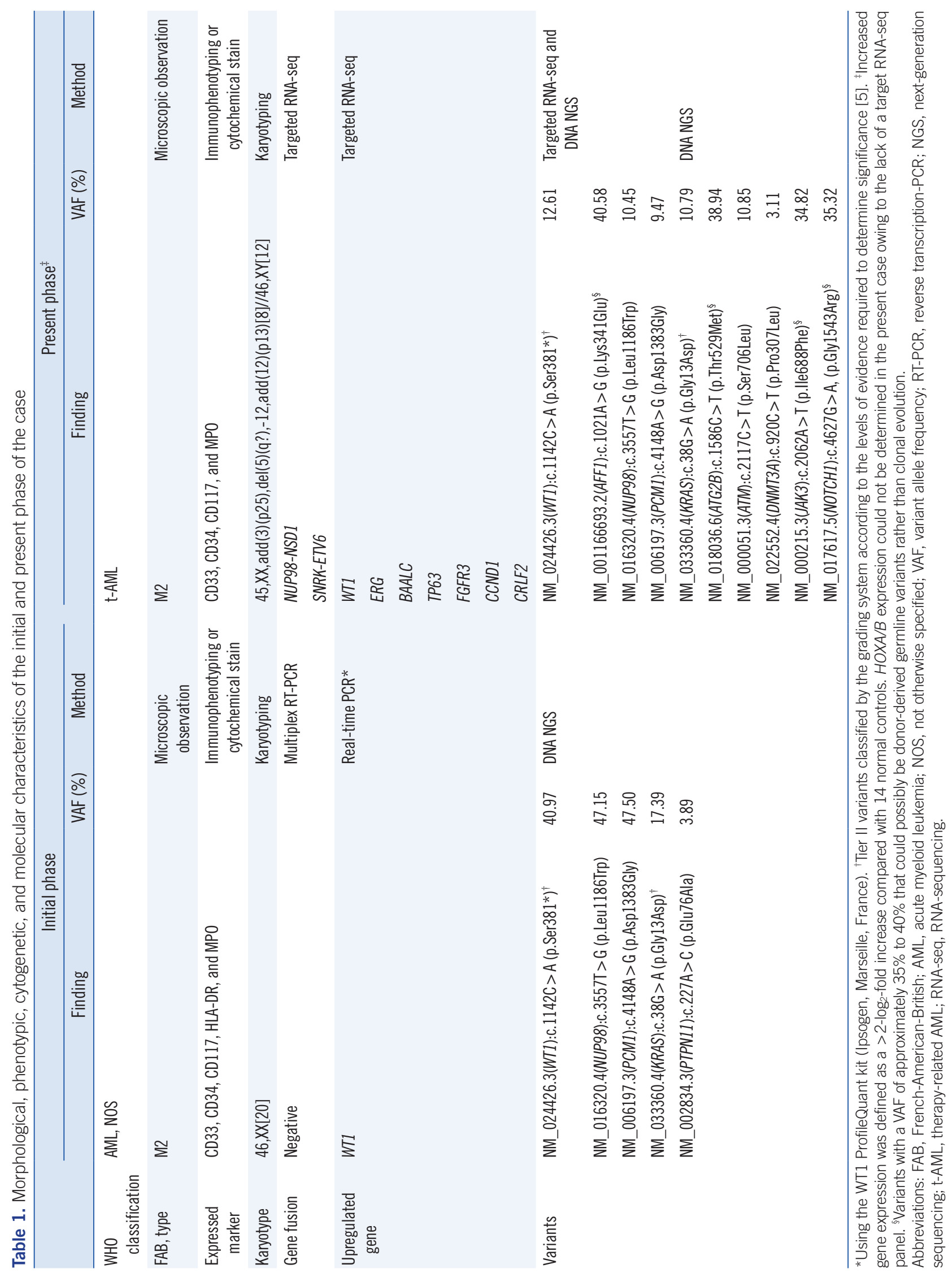


the retrospective nature of this study, the NUP98-NSD1 and SNRK-ETV6 status at the initial diagnostic phase could not be ascertained.

Compared with previous studies using multiple diagnostic methods to characterize NUP98-NSD1+ AML [2], the advantage of the present case was the use of RNA-seq, representing a simplified diagnostic step for gene fusion, expression, and gene variant analyses. Additionally, this system might help uncover novel genetic characteristics in leukemias in future larger-scale studies.

\section{AUTHOR CONTRIBUTIONS}

Lim HJ and Lee JH conceived and designed the study and collected and analyzed the data; Baek $\mathrm{HJ}$ and Kook $\mathrm{H}$ contributed to the data; Lim HJ and Shin MG wrote the final manuscript; Lee YE, Park JH, Lee SY, Choi HW, Choi HJ, Kee SJ, and Shin $\mathrm{JH}$ participated in coordination and discussion. All authors have accepted their responsibility for the entire content of this manuscript and approved the submission.

\section{CONFLICTS OF INTEREST}

No potential conflicts of interest relevant to this article were reported.

\section{RESEARCH FUNDING}

This research was supported by the National Research Foundation of Korea (NRF) grant funded by the Ministry of Science and ICT (MSIT) and the Ministry of Health and Welfare (MOHW) (NRF-2019M3E5D1A02067952).

\section{ORCID}

Ha Jin Lim Jun Hyung Lee Young Eun Lee Hee-Jo Baek Hoon Kook
Ju Heon Park

Seung Yeob Lee Hyun-Woo Choi

Hyun-Jung Choi

Seung-Jung Kee

Myung Geun Shin
Jong Hee Shin https://orcid.org/0000-0002-6842-0234

https://orcid.org/0000-0003-2851-1112

https://orcid.org/0000-0002-9438-1603 https://orcid.org/0000-0003-2901-5962 https://orcid.org/0000-0001-9708-5837 https://orcid.org/0000-0001-9593-476X https://orcid.org/0000-0002-0372-9185

\section{REFERENCES}

1. Zhong Y, Xu F, Wu J, Schubert J, Li MM. Application of next generation sequencing in laboratory medicine. Ann Lab Med 2021;41:25-43.

2. Hollink IH, van den Heuvel-Eibrink MM, Arentsen-Peters ST, Pratcorona M, Abbas S, Kuipers JE, et al. NUP98/NSD1 characterizes a novel poor prognostic group in acute myeloid leukemia with a distinct HOX gene expression pattern. Blood 2011;118:3645-56.

3. Niktoreh N, Walter C, Zimmermann M, von Neuhoff C, von Neuhoff N, Rasche M, et al. Mutated WT1, FLT3-ITD, and NUP98-NSD1 fusion in various combinations define a poor prognostic group in pediatric acute myeloid leukemia. J Oncol 2019;2019:1609128.

4. Franks TM, McCloskey A, Shokirev MN, Benner C, Rathore A, Hetzer MW. Nup98 recruits the Wdr82-Set1A/COMPASS complex to promoters to regulate $\mathrm{H} 3 \mathrm{~K} 4$ trimethylation in hematopoietic progenitor cells. Genes Dev 2017;31:2222-34.

5. Li MM, Datto M, Duncavage EJ, Kulkarni S, Lindeman NI, Roy S, et al. Standards and guidelines for the interpretation and reporting of sequence variants in cancer: a joint consensus recommendation of the Association for Molecular Pathology, American Society of Clinical Oncology, and College of American Pathologists. J Mol Diagn 2017;19:4-23.

6. Kivioja JL, Thanasopoulou A, Kumar A, Kontro M, Yadav B, Majumder MM, et al. Dasatinib and navitoclax act synergistically to target NUP98NSD1(+)/FLT3-ITD(+) acute myeloid leukemia. Leukemia 2019;33:136072.

7. Mitani Y, Hiwatari M, Seki M, Hangai M, Takita J. Successful treatment of acute myeloid leukemia co-expressing NUP98/NSD1 and FLT3/ITD with preemptive donor lymphocyte infusions. Int J Hematol 2019;110: 512-6.

8. De Braekeleer E, Douet-Guilbert N, Morel F, Le Bris MJ, Basinko A, De Braekeleer M. ETV6 fusion genes in hematological malignancies: a review. Leuk Res 2012;36:945-61.

9. Kertesz N, Samson J, Debacker C, Wu H, Labastie MC. Cloning and characterization of human and mouse SNRK sucrose non-fermenting protein (SNF-1)-related kinases. Gene 2002;294:13-24.

10. Block AW, Carroll AJ, Hagemeijer A, Michaux L, van Lom K, Olney HJ, et al. Rare recurring balanced chromosome abnormalities in therapyrelated myelodysplastic syndromes and acute leukemia: report from an international workshop. Genes Chromosomes Cancer 2002;33:401-12. 